\title{
BERNSTEIN FUNCTIONS OF SEVERAL SEMIGROUP GENERATORS ON BANACH SPACES UNDER BOUNDED PERTURBATIONS
}

\author{
A. R. MiRotin
}

Abstract. The paper deals with (multidimensional and one-dimensional) Bochner-Phillips functional calculus. Bounded perturbations of Bernstein functions of (one or several commuting) semigroup generators on Banach spaces are considered, conditions for Lipschitzness and Frechetdifferentiability of such functions are obtained, estimates for the norm of commutators are proved, and a generalization of Livschits-Krĕ̌n trace formula derived.

Mathematics subject classification (2010): 47A56, 47B10, 47L20.

Keywords and phrases: Bochner-Phillips functional calculus, Frechet-differentiable function, semigroup of operators, operator Lipschitz function, trace formula, perturbation of an operator.

\section{REFERENCES}

[1] A. B. Aleksandrov, V. V. Peller, D. Potapov, And F. Sukochev, Functions of normal operators under perturbations, Advances in Math. 226 (2011), 5216-5251.

[2] A. B. Aleksandrov, F. L. Nazarov, V. V. Peller, Functions of noncommuting self-adjoint operators under perturbation and estimates of triple operator integrals, May 27, 2015. Preprint, arXiv:1505.07173v1 [math. FA].

[3] D. Applebaum, Levy Processes - From Probability to Finance and Quantum Groups, Notices of the Amer. Math. Soc. 51, 11 (2004), 1336-1347.

[4] B. Baeumer, M. Kovacs And M. M. Meerschaert, Subordinated Multiparameter Groups of Linear Operators: Properties via the Transference Principle, H. Amann, W. Arendt, M. Hieber, F. Neubrander, S. Nicaise, J. von Below (eds.), Functional Analysis and Evolution Equations. The Gunter Lumer Volume, Birkhauser Verlag, Basel/Switzerland, (2007) 35-50.

[5] C. Berg, K. Boyadzhiev, R. DE LAubenfels, Generation of generators of holomorphic semigroups, J. Austral. Math. Soc. (Series A), 55 (1993), 246-269.

[6] Ch. Berg, J. P. R. Christensen, P. Ressel, Harmonic analysis on semigroups, Grad. Texts in Math., vol. 100, Springer-Verlag, New York-Berlin, 1984.

[7] M. S. BIRMAN AND D. R. YAFAEV, The spectral shift function. The papers of M. G. Krĕ̌n and their further development, Algebra i Analiz 4 (1992), 1-44 (Russian), English transl.: St. Petersburg Math. J. 4 (1993), 833-870.

[8] S. BochneR, Harmonic analysis and the theory of probabylity, University of California Press, Berkeley and Los Angeles, 1955.

[9] A. S. Carasso, T. Kato, On subordinated holomorphic semigroups, Trans. Amer. Math. Soc., 327 (1991), 867-878.

[10] YU. L. DALETSKII AND S. G. KREǏN, Integration and differentiation of functions of Hermitian operators and application to the theory of perturbations (Russian), Trudy Sem. Functsion. Anal., Voronezh. Gos. Univ. 1 (1956), 81-105.

[11] A. Defant And K. Floret, Tensor norms and operator ideals, North-Holland, Amsterdam, 1993.

[12] W. Feller, An Introduction to Probability Theory and Its Applications, 2, Wiley, New York, 1971.

[13] E. Hille And R. Phillips, Functional Analysis and Semigroups, Amer. Math. Soc., Providence, R. I., 1957.

[14] E. Kissin, V. Lomonosov, Tu. TurovskiI, Victor Shulman: The Gentle Art of Mathematics, Operator Theory: Advances and Applications, 233 (2013), 1-16. 
[15] E. Kissin, V. S. Shulman, Classes of operator-smooth functions, III, Stable functions and Fuglede ideals, Proc. Edinburgh Math. Soc. 48 (2005), 175-197.

[16] E. Kissin, V. S. Shulman, Classes of operator-smooth functions, I, Operator Lipschitz functions, Proc. Edinburgh Math. Soc. 48 (2005), 151-173.

[17] E. Kissin, D. Potapov, F. Sukochev, And V. S. Shulman, Lipschitz functions, Schatten ideals and unbounded derivations, Functional Analysis and its Applications 45, 2 (2011), 93-96.

[18] A. Kishimoto, D. Robinson, Subordinate semigroups and order properties, J. Austral. Math. Soc. (Series A), 31, 1 (1981), 59 - 76.

[19] M. G. KREǏN, On a trace formula in perturbation theory, Mat. Sbornik 33 (1953), 597-626 (Russian).

[20] I. M. LIFSHITZ, On a problem in perturbation theory connected with quantum statistics, Uspekhi Mat. Nauk 7 (1952), 171-180 (Russian).

[21] A. R. Mirotin, On the $\mathscr{T}$-calculus of generators for $C_{0}$-semigroups, Sib. Matem. Zh., 39, 3 (1998), 571-582; English transl.: Sib. Math. J., 39, 3 (1998), 493-503.

[22] A. R. Mirotin, Criteria for Analyticity of Subordinate Semigroups, Semigroup Forum, 78, 2 (2009), 262-275.

[23] A. R. Mirotin, Functions from the Schoenberg class $\mathscr{T}$ on the cone of dissipative elements of a Banach algebra, Mat. Zametki, 61, 4 (1997), 630-633; English transl.: Math. Notes, 61, 3-4 (1997), 524-527.

[24] A. R. Mirotin, Functions from the Schoenberg class $\mathscr{T}$ act in the cone of dissipative elements of a Banach algebra, II, Mat. Zametki, 64, 3 (1998), 423-430; English transl.: Math. Notes, 64, 3-4 (1998), 364-370.

[25] A. R. Mirotin, Multidimensional $\mathscr{T}$-calculus for generators of $C_{0}$ semigroups, Algebra i Analiz, 11, 2 (1999), 142-170; English transl.: St. Petersburg Math. J., 11, 2 (1999), 315-335.

[26] A. R. Mirotin, On some properties of the multidimensional Bochner-Phillips functional calculus, Sib. Mat. Zhurnal, 52, 6 (2011), p. 1300-1312; English transl.: Siberian Mathematical Journal 52, 6 (2011), pp. 1032-1041.

[27] A. R. Mirotin, On joint spectra of families of unbounded operators, Izvestiya RAN: Ser. Mat., 79, 6 (2015), 145-170; English transl.: Izvestiya: Mathematics, 79, 6 (2015), 1235-1259.

[28] A. R. Mirotin, Properties of Bernstein functions of several complex variables, Mat. Zametki, 93, 2 (2013), 257-265; English transl.: Math. Notes, 93, 2 (2013).

[29] A. R. Mirotin, On multidimensional Bochner-Phillips functional calculus, Probl. Fiz. Mat. Tekh., 1, 1 (2009), 63-66 (Russian).

[30] S. N. NАBOKO, Estimates in operator classes for a difference of functions, from the Pick class, of accretive operators, Functional Analysis and Its Applications, 24, 3 (1990), 187-195.

[31] A. PAZY, Semigroups of linear operators and applications to partial differential equations, SpringerVerlag, New York-Berlin, 1983.

[32] V. V. PELLER, The behavior of functions of operators under perturbations, April 10, 2009, preprint, http://arxiv.org/abs/0904.1761 [math. FA].

[33] V. V. PelleR, The Lifshitz-Krein trace formula and operator Lipschitz functions, Proc. Amer. Math. Soc. Published electronically: August 1, 2016. DOI: http://dx.doi.org/10.1090/proc/13140.

[34] J. RozendaAl, F. SuKochev, AND A. Toms Kova, Operator Lipschitz functions on Banach spaces, Studia Mathematica 232, 1 (2016), 57-92.

[35] K. Sato, Levi processes and infinitely divisible distributions, Cambridge University Press, Cambridge, 1999.

[36] R. Shilling, R. Song, Z. VondraceK, Bernstein functions. Theory and applications, de Greyter, Berlin-New York, 2010.

[37] V. S. Vladimirov, Yu. N. Drozhzhinov, B. I. Zavialov, Multidimensional Tauberian theorems for generalized functions, Nauka, Moscow, 1985 (Russian).

[38] J. VoIGT, On the convex compactness property for the strong operator topology, Note Mat., 12 (1992), 259-269. 